\title{
PROMOÇÃO DE VENDAS: UMA ANÁLISE DOS FATORES INFLUENGCIADORES DA INTENÇÃO DE COMPRA DE UM PRODUTO EM DESCONTO
}

\author{
ANALYSIS OF THE EFFECTS OF DISCOUNT SALES PROMOTION IN PURCHASE INTENT
}

\author{
Recebido em 19.09.2014. Aprovado em 27.10.2015 \\ Avaliado pelo sistema double blind review \\ DOI: http://dx.doi.org/10.12712/rpca.v9i4.490
}

\section{Fernando Oliveira Santini}

santiniconsultores@terra.com.br

Pontíficia Universidade Católica do Rio Grande do Sul (PUC-RS), PortoAlegre/RS, BRASIL

\section{Rafael Mendes Lübeck}

rafael.lubeck@gmail.com

Pontíficia Universidade Católica do Rio Grande do Sul (PUC-RS), PortoAlegre/RS, BRASIL

\section{Cláudio Hoffmann Sampaio}

csampaio@pucrs.br

Pontíficia Universidade Católica do Rio Grande do Sul (PUC-RS), PortoAlegre/RS, BRASIL

\section{Resumo}

A pesquisa se propôs a avaliar o efeito da promoção de vendas de desconto sobre a intenção de compra. Investigou-se como os comportamentos associados à atratividade da campanha, impulsividade, percepção hedônica e risco financeiro se relacionam com o produto com desconto. Foi realizado um experimento de laboratório junto a 670 alunos de graduação do curso de administração de empresas de uma Instituição de Ensino Superior localizada no sul do Brasil. Os resultados apresentaram influência significativa da promoção com desconto sobre a intenção de compra do consumidor, especificamente da relação positiva e significativa da atratividade da promoção, impulsividade e percepção hedônica e a relação negativa entre a percepção de risco financeiro e a intenção de compra. Entre as implicações da presente pesquisa destacam-se a reafirmação empírica da eficácia da promoção de desconto na intenção de compra em consumidores brasileiros. No contexto nacional a ocorrência de pesquisas sobre o tema ainda é um desafio e ampliar as pesquisas pode trazer, além de contribuições acadêmicas, contribuições para os varejistas brasileiros compreenderem melhor que tipo de promoção de vendas sensibiliza o consumidor brasileiro.

Palavras-chave: Promoção de Vendas. Promoção de Desconto. Intenção de Compra.

\begin{abstract}
The research aims to evaluate the effect of promoting sales discount on the purchase intent. He intended to investigate how the behaviors associated with the attractiveness of the campaign, impulsivity, hedonic perception and financial risk relate to the product at a discount. A laboratory experiment was conducted among the hundred and seventy undergraduate students of business administration program at a higher education institution located in southern Brazil. The results showed a significant influence of promotional discount on consumer purchase intent specifically the positive and significant relationship of the attractiveness of the promotion, impulsivity, hedonic perception and negative relationship between perceived financial risk and purchase intent. Among the implications of this survey highlight the empirical reaffirming the effectiveness of the discount promotion in purchase intent among Brazilian consumers. In the national context the occurrence of research on the subject will still challenging to bring, in addition to academic contributions, contributions to Brazilian retailers better understand what kind of sales promotion sensitizes the Brazilian consumer.
\end{abstract}

Keywords: Sales Promotion. Product Discount. Purchase Intention. 


\section{Introdução}

As promoções de vendas são atividades que objetivam incrementar o volume de vendas de produtos e serviços num curto espaço de tempo (WIERENGA; SOETHOUDT, 2010), pois produzem efeitos na mente do consumidor, que as interpretam como um benefício e geram um comportamento de consumo (YUSUF, 2010). Os estudos sobre promoção de vendas vêm sendo realizado há algumas décadas, como atestam análises de 1978, produzidas por Preston, Dwyer e Rodelius, que já demonstravam o efeito desta ferramenta promocional no convencimento de consumidores para abrirem uma conta bancária. Recentemente, pesquisas demonstram a eficiência da promoção de vendas na intenção de compra dos consumidores em relação a computadores (D’ASTOUS; JACOB, 2002) e na adesão a serviços financeiros (BOSCHET'TI, 2012).

$\mathrm{Na}$ esfera gerencial, pesquisas demonstram a importância dessa ferramenta promocional nas estratégias de marketing das empresas. Em 2002, Teunter atestava que mais de $20 \%$ das vendas de determinada categoria de produto eram influenciados por ações de promoção de vendas. Em 2010, Wierenga e Soethoudt relatavam que mais de $75 \%$ dos gastos com marketing no segmento de bens de consumo não duráveis, entre 1997 e 2004, nos Estados Unidos, foram direcionados à promoção de vendas.

No âmbito acadêmico há incentivo a investigações que busquem o entendimento de influências potencializadoras do comportamento associadas à promoção de vendas, como é o caso da percepção de atratividade (p. ex. ALVAREZ; CASIELLES, 2005), assim como para investigar variáveis de marketing que possam estimular ou minimizar um comportamento impulsivo (p. ex. MIHIK; KURSAN, 2010), consumo hedônico (p. ex. HUDSON, 2007), percepção de risco sobre um produto (p. ex. APADYN; KOKSAL, 2011).

Assim, o estudo está alinhado à busca de conhecimento sobre o impacto da promoção de vendas, especialmente no ambiente brasileiro, onde as publicações sobre o tema são incipientes (SANTINI, 2013) e, particularmente, no segmento de varejo, no qual as ações de promoção de vendas e de desconto são amplamente utilizadas (OH; KNOW, 2008; TONG; LAI; TONG, 2011; SANTINI, 2013).

Diante desse contexto, busca-se preencher as lacunas já citadas fomentando a discussão de modo a contribuir ao entendimento do efeito da promoção de vendas de desconto na intenção de compra dos consumidores, avaliando também como os comportamentos de atratividade (SIMONSON; CARMON; O'CURRY, 1994; D'ASTOUS; LANDREVILLE, 2003; LIAO, 2006, BOSCHETTI, 2012), de impulsividade (ROOK, 1987, ROOK; FISHER, 1995; PURI, 1996; RAMANATHAN; MENON, 2006), de percepção hedônica do produto (HIRSCHMAN; HOLDBROOK, 1982; BATRA; AHTOLA, 1990; CARDOSO; PINTO, 2010) e também de risco financeiro (BAWA; SHOEMAKER, 1987; LATTIN; BUCKLIN, 1989; BLATTBERG; NESLIN, 1990; ALVAREZ; CASIELLES, 2005) afetarão a intenção de compra de um produto em desconto. Para tanto, realizou-se uma pesquisa experimental de laboratório, com a participação de seiscentos e setenta alunos de um curso de graduação de uma Instituição de Ensino Superior.

Espera-se visualizar nos resultados que a promoção de desconto, a atratividade, a percepção hedônica do produto e a impulsividade aumentem a intenção de compra do produto anunciado e que quanto menor for o risco financeiro maior será a intenção de compra. Para isso testou-se as hipóteses descritas a seguir.

\section{Reflexões teóricas e hipóteses da pesquisa}

Promoção de vendas consiste em uma ferramenta de marketing utilizada para estimular o consumo de produtos e serviços (D'ASTOUS; LANDREVILLE, 2003; LIAO, 2006). Tal estratégia tem como objetivo influenciar o processo de escolha do consumidor $\mathrm{e}$ busca prover atração e valor aos produtos ofertados (BLATTBERG; NESLIN, 1990; KELLER, 1993; ALVAREZ; CASIELLES, 2005). As técnicas de promoção de vendas podem ser separadas em dois grupos distintos: a) promoções voltadas para preço, ou monetárias (descontos, cupons, abatimento); b) promoções não voltadas para preço, ou não monetárias (amostras grátis, promoção de distribuição de prêmios, concursos e programas de fidelidade).

As técnicas de promoções voltadas para preço são atividades que buscam obter resultados em curto prazo (BAWA; SHOEMAKER, 1987; BLATT'BERG; NESLIN, 1990), encorajamento de troca de marca (GUPTA, 1988; DAVIS; INMAM; MCALISTER, 1992) e indução à experiência de uso (LEE, 2002), 
enquanto a promoção não voltada para preço é adequada para ações de longo prazo, como promoção de imagem da marca, ações estratégicas ou aumento do marketshare (CONLON, 1980; JAGODA, 1984; AAKER, 1991). Para o caso deste estudo, optou-se em avaliar a promoção de cárter monetário, sendo selecionada para análise a técnica de promoção de desconto.

As promoções voltadas para preços são referenciadas como melhor alternativa na busca de venda em curto prazo, já que esta variável é fundamental na decisão de escolha de um consumidor (WINER, 1986; LAT'TIN; BUCKLIN, 1989; ALVAREZ; CASIELLES, 2005). Blattberg e Neslin (1990) assumem que esse tipo de promoção vai ao encontro da motivação de economia dos consumidores. Alguns estudos apontam que os consumidores sempre dão respostas a campanhas de desconto (DAVIS; INMAM; MCALISTER,1992; NIJS et al., 2001; TAYLOR; NESLIN, 2005). Promoções voltadas para preços são baseadas em incentivos transacionais, que proporcionam recompensas imediatas e benefícios utilitários (CHANDON; WANSINK; LAURENT, 2000; KWOK; UNCLES, 2005). Para Tan e Chua (2004), essa técnica é avaliada pelos consumidores como uma redução de perda. Com base neste contexto, entendeu-se que a promoção de desconto sensibiliza o consumidor influenciando diretamente em sua escolha. Ante um desconto entende-se que o consumidor ao perceber que terá uma vantagem financeira ficará mais propenso a compra desse produto devido a estar recebendo um benefício além do que o produto já oferece, um algo mais que poderá aumentar sua propensão a aquisição daquele item naquele momento. Com isso, propõe-se a primeira hipótese a ser testada:

H1: A promoção vendas de desconto irá influenciar positivamente a intenção de compra de um produto.

Considerando a proposição exposta na primeira hipótese, acredita-se que a intenção de compra da promoção de desconto pode ser motivada pela percepção da atratividade da campanha promocional, já que este sentimento é ressaltado por diversos estudos como fator norteador do sucesso ou fracasso de uma promoção (SIMONSON; CARMON; O'CURRY, 1994; D'ASTOUS; LANDREVILLE, 2003; LIAO, 2006).
D'Astous e Jacob (2002) e D'Astous e Landreville (2003) ratificam o pressuposto de que a percepção da atratividade da promoção é uma variável significativa para explicar uma avaliação positiva sobre um produto ou marca ofertada. Laroche et al. (2003) detectaram, também, que o interesse do consumidor frente à promoção é correlacionado com a intenção de compra do produto em oferta. Reforçando esta suposição, Liao (2006) identificou, em seu estudo, que o tipo de prêmio distribuído tem papel fundamental para a ação realizada. Palazón e Delgado-Ballester (2009) investigaram a interação da atratividade da promoção entre campanhas monetárias e não monetárias. No âmbito monetário, os resultados demonstram que a promoção atrativa de desconto impacta positivamente na intenção de compra, enquanto a de atratividade baixa tem menos efetividade neste comportamento.

Diante das informações postuladas e considerando-se o efeito positivo que a atratividade da promoção tende a exercer sobre os comportamentos associados a uma campanha, espera-se que a atratividade do prêmio interfira positivamente na intenção de compra de um produto em desconto, assim:

H2: A atratividade da campanha promocional irá influenciar positivamente a intenção de compra do produto em desconto.

Outra suposição proposta nesta pesquisa é a relação entre a impulsividade e a intenção de comprar um produto em desconto. A impulsividade está associada a componentes afetivos e hedônicos da personalidade (WEINBERG; GOTTWALD, 1982; ROOK, 1987; ROOK; FISHER, 1995, YOUN, 2000). Coley e Burgess (2003), em raciocínio próximo aos dos autores citados, relacionam o comportamento impulsivo a seis componentes principais: a) propensão irresistível à compra; b) emoção positiva relacionada à compra; c) gerenciamento do humor; d) decisão cognitiva; e) compra não planejada; f) desconsideração em relação ao futuro.

$\mathrm{Na}$ presente pesquisa, pressupõe-se que a impulsividade do consumidor irá interagir de forma positiva com a intenção de comprar produtos em desconto, uma vez que é detectada relação entre satisfação de curto prazo dos consumidores impulsivos (DHOLAKIA; 2000; YOUN; FABER, 2000) e os benefícios proporcionados aos consumidores que 
adquirem produtos sob os efeitos das promoções monetárias - caso das campanhas de descontos, que têm o impacto imediato, ou seja, também efeito de curto prazo (WINER, 1986; BAWA; SHOEMAKER, 1987; LATTIN; BUCKLIN, 1989; BLATTBERG; NESLIN, 1990; ALVAREZ; CASIELLES, 2005).

Devido aos argumentos apresentados compreende-se que no momento em que o consumidor não racionaliza a compra de um produto, não leva em conta, por exemplo, sua capacidade de pagar pelo produto ou mesmo se esse terá utilidade. Consumidores mais impulsivos diante de um estímulo como o desconto deverão ser mais sensíveis a adquirir o produto anunciado, por essas razões, surge a hipótese a seguir:

H3: A impulsividade do consumidor irá influenciar positivamente a intenção de compra do produto em desconto.

Seguindo as proposições do estudo, vislumbra-se que exista uma relação entre percepção hedônica do produto ofertado e intenção de compra em desconto. O conceito de consumo hedônico foi introduzido por Hirschman e Holbrook (1982), quando relacionaram esse construto ao envolvimento multissensorial e emotivo que, por sua vez, está vinculado às experiências dos consumidores e aos produtos. A dimensão de consumo hedônico pode ser derivada da exclusividade do bem, do seu significado simbólico, da excitação emocional e da evocação imaginária (HIRSCHMAN; HOLBROOK, 1982; SPANGENBERG; VOSS; CROWLEY, 1997). Essa dimensão é mais subjetiva e pessoal do que a utilitária (BABIN; DARDEN; GRIFFIN, 1994). A motivação do consumo hedônico é derivada de uma necessidade de prazer e excitação, que podem ser proporcionadas por esses produtos (DHAR; WERTENBROCH, 2000). Os caminhos do prazer são as reais motivações do consumo hedônico (RAMANATHAN; MENON, 2006).

Estudos demonstram que não é somente o ganho financeiro que está atrelado à promoção de vendas (INMAN; MCALISTER; HOYER, 1990; SCHINDLER, 1992; HOCH; DRĖZE; PURK, 1994; DHAR; HOCH, 1996). Isso sugere que efeitos emocionais e, nesse caso, o benefício hedônico, também podem ajudar a explicar, por exemplo, por que consumidores respondem com mais força a campanhas de cupons em lojas do que a propagandas que anunciam a redução de preço (SCHINDLER, 1992; DHAR; HOCH, 1996) e, ainda, por que consumidores respondem à redução insignificante de preço (INMAN; MCALISTER; HOYER, 1990; HOCH; DRĖZE; PURK, 1994). Segundo Babin, Darden e Griffin (1994), os benefícios hedônicos obtidos por meio da percepção de barganha ou desconto aumentam o envolvimento sensorial e de excitação. Compras de valor também podem estar relacionadas à dimensão de otimização de escolhas, proposta por Westbrook e Black (1985), já que o fato de encontrar um desconto ou uma barganha pode levar à satisfação da realização pessoal, característica do consumo hedonista (HIRSCHMAN; HOLBROOK, 1982; SPANGENBERG; VOSS; CROWLEY, 1997). Consumo hedônico se efetiva pela sensibilização emocional que o produto causa no consumidor e a partir do momento que o consumidor percebe um benefício hedônico do produto associado a um forte estímulo como o desconto espera-se que a intenção de compra seja positiva. Diante dessas constatações sugere-se a hipótese a seguir:

H4: A percepção hedônica do produto irá influenciar positivamente a intenção de compra do bem em desconto.

Por fim, aguarda-se uma relação existente entre percepção de risco financeiro e intenção de compra de um produto em desconto. Não só no campo do marketing, mas também na área da psicologia, o debate sobre este tema tem se destacado (MITCHELL, 1999; DHOLAKIA, 2000). Dholakia (2000) estabelece o conceito geral de risco percebido como um sentimento negativo que poderá ser gerado por meio da aquisição de um produto/serviço. Diversos estudos demonstram que o sentimento de risco percebido afeta a propensão do consumidor a tomar sua decisão no momento da compra (HISRICH; DORNOFF; KERNAN, 1972; JASPER; OUELLETTE, 1994; SITKIN; WEINGART, 1995; CHOI; LEE, 2003). Nesse sentido, e no contexto da teoria de risco, as pessoas, ao terem consciência deste sentimento, avaliam o risco antes de tomar uma decisão e o sentimento de risco é aumentado em situações que fogem ao cotidiano. A falta do conhecimento prévio é influenciada por uma série de fatores e potencializa a sensação de risco (WEEGELS; KANIS, 2000). 
Uma das dimensões do risco percebido associa-se ao risco financeiro, e ocorre quando o sentimento do consumidor vincula-se à perda monetária que o bem a ser adquirido pode acarretar (JACOBY; KAPLAN, 1972; DHOLAKIA, 2000; HUANG; SCHRANK; DUBINSKY, 2004). Essa perda poderá estar atrelada a uma simples desvalorização do produto ou a gastos com consertos e manutenção (MITCHELL, 1999; APAYDIN; KÖKSAL, 2011) e é uma das dimensões mais significativas na determinação desse sentimento (OFIR; BECHTEL, 1990; MITCHELL, 1999; AGARWAL; TEAS, 2001). Na mesma linha, estudos são realizados para analisar especificamente essa dimensão do risco percebido (SIMON; VICTOR, 1994; DIACON, 2004; ENGELBERG, 2007).

Nesse contexto, surge a primeira relação que pode estar associada à promoção de vendas e a uma força maior para ações de campanhas com objetivo monetário como, por exemplo, desconto, já que esta ferramenta objetiva reduzir o valor tradicional do produto ofertado (WINER, 1985; BAWA; SHOEMAKER, 1987; BLATTBERG; NESLIN, 1990; GUPTA, 1988; LEE, 2002; ALVAREZ; CASIELLES, 2005). Essa relação é explicitada por Tan e Chua (2004), quando dizem que as promoções monetárias são avaliadas pelos consumidores como uma redução de perda.

Da mesma forma, Simon e Victor (1994) afirmam que os incentivos monetários são alternativas eficientes para os consumidores diminuírem a percepção de risco. Essa vinculação fica ainda mais clara quando, no estudo de Garretson, Burton e Clow (1999), é testada e confirmada hipótese que propõe a utilização de cupons de desconto na diminuição da percepção de risco associada ao consumo de um serviço.

Este estudo utilizou a dimensão de risco financeiro e levando em consideração que este sentimento está atrelado aos aspectos monetários de um produto entende-se que ao perceber que um produto com desconto representa risco financeiro o consumidor tende a protejer-se desse risco minimizando sua intenção de compra. Assim apresenta-se a hipótese a seguir:

H5: A percepção de risco financeiro irá influenciar negativamente a intenção de compra do bem em desconto.

\section{Procedimentos metodológicos}

Em face dos objetivos traçados para este estudo foi realizada uma pesquisa de natureza experimental precedida de etapa exploratória e descritiva que objetivou definir o produto e desconto a ser utilizado na etapa experimental. A pesquisa experimental é indicada para testar relações de causa e efeito (MALHOTRA, 2006) - caso deste estudo. Os experimentos fornecem maior controle aos pesquisadores, sendo capazes de fornecer evidências mais convincentes de relações causais do que estudos exploratórios ou descritivos (CHURCHILL, 1999). Existe um experimento quando se manipula uma ou mais variáveis independentes e se mede o seu efeito sobre uma ou mais variáveis dependentes, ao mesmo tempo em que se controla o efeito de variáveis estranhas. O objetivo é verificar se há variáveis dependentes à medida que se manipulam as variáveis independentes ou fatores (MALHOTRA, 2006).

\section{Desenvolvimento do instrumento de coleta dos dados}

O questionário utilizado nesta pesquisa buscou obter medir as reações dos respondentes em dois cenários (sem desconto e com desconto) sobre: a) intenção de compra do produto obtida a partir da escala de Bruner e Hensel (1998); percepção hedônica do produto (VOSS; SPANGENBERG; GROHMANN, 2003); risco financeiro em relação ao produto ofertado (STONE; GRONBAUG, 1993); impulsividade (PURI, 1996) e atratividade da promoção (D’AUSTOUS; LANDREVILLE, 2003).

Realizada uma primeira versão do questionário o mesmo foi submetido à validação de conteúdo, realizada por três doutores em administração e a um pré-teste, junto 20 alunos de graduação do público alvo da pesquisa. A validação de conteúdo demandou pequenos ajustes na formatação do questionário, enquanto que o pré-teste não apresentou nenhum problema em relação ao entendimento das questões utilizadas e a familiaridade com produto utilizado para o experimento. 


\section{Preparação do experimento realizado: desenvolvimento dos cenários}

Conforme mencionado, foi realizada uma pesquisa de natureza exploratória para a definição de quais seriam os preços e os descontos atraentes ao respondente, cujo objetivo foi a preparação dos cenários para o experimento realizado neste estudo. A etapa exploratória norteou a identificação de possíveis produtos e descontos alinhados com a realidade do público participante do estudo.

Nessa fase, a coleta de informações se deu por meio de dados secundários - no site de compras coletivas Groupon, de onde foram analisados os produtos e descontos ofertados durante o período de 02 a 17 de julho de 2012. A partir da efetivação desta análise, foi possível avaliar os dez produtos mais frequentes e os descontos no período considerado.

Uma vez realizada a identificação dos produtos e descontos obtidos na etapa exploratória, chegou-se na etapa descritiva onde culminou na consolidação do produto mais familiar e realista a vista dos participantes, uma vez que esta iniciativa é essencial para validade interna e externa de um experimento de laboratório (WILSON; ARONSON; CARLSMITH, 2010).

Antes da aplicação do instrumento de pesquisa da etapa descritiva, foi realizada a validação de conteúdo do questionário além de pré-teste. $\mathrm{Na}$ validação de conteúdo, realizada por três Doutores da área da Administração, foram sugeridas a exclusão de três produtos, por não estarem alinhadas com a realidade dos entrevistados (luminária; lavadora de alta pressão e; aliança) além do produto "vídeo game" por ser um bem que remete a uma marca, como por exemplo: X Box e PlayStation. O pré-teste realizado com 15 (quinze) estudantes, os quais responderam questionários sobre diferentes tipos de produtos, não demandaram ajustes nas escalas utilizadas e tampouco a reavaliação dos produtos anunciados.

Chegou-se para aplicação da etapa descritiva, em seis diferentes produtos aplicados individualmente, num instrumento de pesquisa que possuíam escalas de: a) interesse sobre o produto de Bruner e Hensel (1998); b) percepção hedônica ou utilitária de Voss, Spangenberg e Grohmann (2003) e; c) risco financeiro de Stone e Gronhaug (1993). Os questionários foram aplicados junto a 182 estudantes do curso de administração de empresas de uma Instituição de Ensino Superior localizada no sul do Brasil. Para cada produto testado, a amostra estipulada foi de, no mínimo, trinta questionários válidos, uma vez que este é o número tolerável para a realização de testes estatísticos efetivados (MANN, 2006 apud PINHEIRO; SILVA; RODRIGUES, 2011).

O resultado apresentado nesta etapa identificou o produto "câmera fotográfica" com o comportamento mais favorável em relação ao interesse, percepção hedônico e utilitário, além da menor percepção de risco e, portanto, foi o escolhido para a etapa experimental. A seguir, a seção segue com a explanação sobre a população e amostra da pesquisa.

Posteriormente desenvolveu-se um experimento de laboratório em que foi manipulado o fator promoção (com promoção de desconto vs. sem promoção de desconto). Optou-se por utilizar o método whitinsubjects, no qual cada participante da amostra é submetido a todas as condições experimentais (KEPPEL, 1991).

Como já mencionado, o fator manipulado foi à promoção (com desconto vs. sem desconto). Para isto, primeiramente era entregue aos pesquisados um anúncio do produto selecionado na etapa anterior. Este anúncio fazia uma breve descrição do produto, com seu valor aproximado, sem incidência do desconto, e trazia cinco perguntas para avaliar a intenção de compra dos respondentes. Estavas variáveis servirão para mensurar o interesse de compra do objeto, sem o efeito da promoção de vendas.

Uma vez preenchido, o questionário era recolhido e o participante recebia novamente o anúncio do mesmo produto, com as mesmas características, porém, agora, com o destaque do percentual da promoção de desconto e o novo valor do produto. Além desta informação, os respondentes eram submetidos às escalas de intenção de compra, percepção hedônica, risco financeiro, impulsividade e atratividade da promoção.

\section{Controle das variáveis estranhas}

As variáveis estranhas constituem-se em um problema para o pesquisador, pois são variáveis que não afetam a independente, mas que podem interferir nos efeitos das dependentes (MALHOTRA, 2006). Em vista 
disso, alguns cuidados foram tomados para evitar os efeitos das variáveis estranhas neste estudo.

Primeiramente, nenhum produto ofertado continha marca. Esta decisão tem como objetivo evitar a influência destas variáveis na intenção de compra do participante, haja vista que outros estudos já encontraram esta relação (KELLER, 1993; AAKER, 1998; KELLER; LEHMANN, 2006), inclusive no Brasil (URDAN; URDAN, 2001). Outra variável controlada foi à influência da lealdade e da confiança associadas à marca sobre a intenção de compra do consumidor (BAWA; SHOEMAMAKER, 1987; LAROCHE et al., 2003; ALVAREZ; CASIELLES, 2005, NAGAR, 2009). Por esta razão, reforça-se a decisão de não utilizar nenhuma marca nos cenários manipulados para controle dessa possível variável estranha.

Outra questão importante controlada em razão da artificialidade do experimento foi o recurso financeiro disponível ao pesquisado, uma vez que esta variável é um elemento influenciador sobre o aumento de incidência de compra (IYER, 1989; HAUSMAN, 2000). Controlaram-se, também, as possíveis influências sociodemográficas nos resultados da pesquisa como, por exemplo, renda, sexo e idade, já que a aplicação de estudos junto a estudantes permite obter certa homogeneidade da amostra e aumento da validade interna do experimento (PETERSON, 2001).

\section{Pré-teste dos cenários}

Antes da aplicação do instrumento de pesquisa, realizaram-se a validação de conteúdo do questionário e o pré-teste. O pré-teste foi realizado com 15 (quinze) estudantes que responderam questionários sobre diferentes tipos de produtos, não demandou ajustes nas escalas utilizadas e tampouco a reavaliação dos produtos anunciados.

Os questionários foram aplicados junto a 182 estudantes do curso de administração de empresas de uma Instituição de Ensino Superior localizada no sul do Brasil. Para cada produto testado, a amostra estipulada foi de, no mínimo, trinta questionários válidos, uma vez que este é o número tolerável para a realização de testes estatísticos efetivos (MANN, 2006).

O resultado apresentado nesta etapa identificou

o produto "câmera fotográfica" como o de comportamento mais favorável em relação ao interesse, percepção hedônica e utilitária, além da menor percepção de risco e, portanto, foi o escolhido para a etapa experimental. A seguir, a seção segue com a explanação sobre a população e amostra da pesquisa.

\section{População e amostra da pesquisa}

A população do estudo consistiu de estudantes do curso de graduação de administração de empresas de uma Instituição de Ensino Superior e amostra foi de seiscentos e noventa e um estudantes, selecionados pelo método de conveniência, que consiste numa seleção não aleatória, caracterizada pela facilidade de acesso do pesquisador aos entrevistados (MALHOTRA, 2012).

Ressalta-se que a amostragem com estudantes é recomendada quando se testa a aplicação de uma teoria, sendo essencial, nestes casos, certa homogeneidade dos respondentes (CALDER; PHILIPS; TYBOUT, 1981). Justifica-se o tamanho da amostra utilizada, por atender com sobra os procedimentos estatísticos aplicados, pois foram utilizadas 45 variáveis sendo que o recomendável é de cinco observações para cada variável (HAIR et al. 2005).

\section{Análise dos resultados}

Uma vez coletados os dados, os mesmos foram tabulados e analisados pela técnica estatística de Teste $t$, através da qual foi possível avaliar a influência da promoção de desconto na intenção de compra do produto ofertado (H1) e, em seguida, as demais análises e testes das hipóteses foram efetivadas pela técnica de Modelagem de Equações Estruturais (MME).

\section{Resultados}

Os dados obtidos na etapa experimental foram tabulados e analisados com o auxílio dos softwares PASW Statistics Data Document 18.0 e AMOS 18.0, sendo os mesmos utilizados para a análise nos procedimentos de estatística descritiva e multivariada. 


\section{Purificação da base de dados}

A purificação da base de dados inicialmente ocorreu a partir da análise dos casos omissos. Para isto, efetivouse a análise de frequência de todas as questões do instrumento de pesquisa, avaliando a possibilidade de alguma delas ter sido mal interpretada. Esta análise não detectou nenhuma situação deste tipo, já que não foi encontrada nenhuma variável com índice de não resposta superior a cinco. Prosseguindo a análise dos casos omissos, avaliou-se se algum elemento da amostra havia não resposta superior a $10 \%$. Nesta etapa identificou onze questionários nesta situação, os quais foram excluídos da amostra final.

Após a identificação dos casos omissos, as análises prosseguiram para verificação dos outliers. Primeiramente, buscou-se a identificação de outliers univariados. Os outliers univariados foram analisados pelo cálculo de Z score, sendo excluídos da amostra final 6 questionários que apresentaram escores superiores a 3 (HAIR et al. 2005). Finalmente, a purificação da base de dados culminou na análise de outliers multivariados, cuja identificação se deu a partir da análise de Mahalanobis, através da qual se verifica a existência de grande distância entre o valor individual e as médias amostrais obtidas (Kline, 1998). Com esta análise, foram identificados quatro elementos, excluídos da amostra final. As etapas seguintes prosseguiram com seiscentos e setenta questionários válidos.

\section{Caracterização da amostra}

Com base no universo total dos questionários válidos, que foram de seiscentos e setenta elementos, efetivouse a análise estatística descritiva para compreender as características amostrais da pesquisa. Sinteticamente, obteve-se uma amostra com uma maior concentração de renda entre $R \$ 1.000$ a $R \$ 3.000$ (53,3\%), com idade entre 19 a 25 anos (52,7\%), do sexo feminino $(52,4 \%)$ e estado civil "solteiro" $(65,2 \%)$.

\section{Discussão dos modelos de medidas}

Os pressupostos de normalidade nas variáveis observadas constituíram a primeira etapa de análise realizada. Com este objetivo, efetivou-se o cálculo dos indicadores de curtose e assimetria. A análise de curtose se deu por meio do Coeficiente de Mardia, sendo detectados valores menores que 5, portanto, significativos, o que indica a presença de normalidade nas distribuições (MARDIA, 1970; BENTLER, 1995). Na análise de assimetria, as variáveis observadas demostraram uma assimetria moderada, já que os coeficientes de assimetria de Pearson foram próximos de zero.

Na sequência, procedeu-se à análise de confiabilidade simples, por meio do Alpha de Cronbach, pela qual fica constatada a consistência interna das escalas utilizadas. Nesta etapa, todas as escalas apresentaram índices superiores ao mínimo tolerável que é de 0,6 (HAIR et al. 2005), sendo apresentado o valor de 0,91 para o construto "intenção de compra sem desconto"; 0,94 para "intenção de compra com desconto"; 0,86 para "percepção hedônica"; 0,76 para "risco financeiro"; 0,64 para "impulsividade" e 0,86 para "atratividade".

Em seguida, efetivou-se a análise fatorial confirmatória, cujo objetivo é ratificar o modelo originalmente proposto na teoria. Os resultados das cargas fatoriais dos itens sobre cada variável latente evidenciou loadings significativos, a 0,01 . Os indicadores de confiabilidade composta e de Variância Média Extraída (AVE) foram considerados significativos. A tabela 1 exibe a validade convergente e discriminante dos construtos utilizados no modelo de mensuração pelo método de Fornell e Larcker (1982).

Tabela 1: Correlações entre os construtos

\begin{tabular}{|c|c|c|c|c|c|c|}
\hline Variável & ISD & ICD & IMP & PH & PR & AT \\
\hline $\begin{array}{l}\text { Intenção de } \\
\text { compra sem } \\
\text { promoção de } \\
\text { desconto (ISD) }\end{array}$ & ,949 & & & & & \\
\hline $\begin{array}{l}\text { Intenção de } \\
\text { compra com } \\
\text { promoção de } \\
\text { desconto (ICD) }\end{array}$ &, 746 & 919 & & & & \\
\hline $\begin{array}{l}\text { Impulsividade } \\
\text { (IMP) }\end{array}$ &, 157 & ,109 & ,678 & & & \\
\hline $\begin{array}{l}\text { Percepção } \\
\text { hedônica }(\mathrm{PH})\end{array}$ &, 121 & ,237 & ,007 &, 868 & & \\
\hline $\begin{array}{l}\text { Percepção de } \\
\text { risco }(\mathrm{PR})\end{array}$ & ,023 &,- 117 & ,057 &, 045 &, 774 & \\
\hline $\begin{array}{l}\text { Atratividade } \\
\text { (AT) }\end{array}$ & ,128 & ,448 &, 040 &, 160 &, 006 & ,863 \\
\hline
\end{tabular}

Nota: Negrito $=$ Raiz quadrada da variância extraída 
Por fim, analisaram-se os índices de ajustamento do modelo integrado, tais como: qui-quadrado/graus de liberdade, normed fit index (NFI), comparative fit index (CFI) e standardired root meansquare residual (RMSEA) (KOUFTEROS, 2009). Os resultados apresentados demonstram índices satisfatórios (ARBUCKLE, 2008; MARÔCO, 2010) para CFI e NFI, 0,960 e 0,939, respectivamente, RMSEA, 0,051 e, por fim, a relação entre qui-quadrado/graus de liberdade foi de 2,76. A seguir, a seção de análise dos resultados prossegue com o teste das hipóteses.

\section{Testes das hipóteses}

A primeira hipótese do estudo previa que a promoção de desconto iria influenciar positivamente a intenção de comprar o produto (neste estudo, uma câmera fotográfica). Para isto, efetivou-se a análise de teste $t$, através da qual realizou-se a comparação geral da média entre as intenções de compra do produto, com a promoção de desconto e sem a promoção de desconto. A análise de teste $t$ avalia as médias entre dois grupos da amostra e verifica se existe diferença estatística entre elas (HAIR et al., 2005) A tabela 2, apresenta o resultado encontrado.

Tabela 2: Diferença das médias entre os construtos "intenção de compra” com e sem desconto

\begin{tabular}{lccccc}
\hline & $\mathbf{n}$ & MÉDIA & DESVIO PADRÃO & SIG & t \\
\hline I. C. sem desconto & 670 & 2,73 & 1,08 & $0,000^{*}$ & $-16,142$ \\
I. C. com desconto & 670 & 3,28 & 1,17 & \\
\hline
\end{tabular}

Fonte: Dados da pesquisa.

(*) médias diferentes estatisticamente significantes; teste t pelo método Paired Samples test t;

I.C = Intenção de Compra.

Os resultados expostos na tabela 1 apresentam uma diferença significativa nas médias dos grupos expostos ao produto com promoção de desconto e sem promoção de desconto. Observa-se que, quando o produto "câmera fotográfica" estava sem a apresentação de desconto, a média de intenção de compra foi de 2.73. Já com a inclusão do desconto esta média passou para 3,28. Dessa forma, os resultados confirmam a proposição da hipótese 1.

Os testes para as hipóteses seguintes foram realizados por meio da Análise de Modelagem de Equações Estruturais. Esta análise foi precedida da validação do modelo (Item 4.3). Após a avaliação do modelo, seguiu-se para a análise do modelo integrado, que agrega o modelo de mensuração e o modelo estrutural, objetivando examinar as relações entre os construtos (intenção de compra do produto em desconto; atratividade; impulsividade; percepção hedônica; risco financeiro) e as variáveis propostas no estudo/modelo. Esta avaliação foi realizada a partir dos índices de ajuste do modelo e de significância estatística dos coeficientes estimados (Kline, 1998). A Tabela 3 apresenta os coeficientes padronizados e significância do modelo integrado.

Tabela 3: Teste de hipóteses (H2 a H5)

\begin{tabular}{|c|c|c|c|c|c|c|}
\hline Hipótese & CONSTRUTOS & $\begin{array}{l}\text { COEFICIENTE } \\
\text { PADRONIZADO }\end{array}$ & $\begin{array}{l}\text { DESVIO } \\
\text { PADRÃO }\end{array}$ & $\bar{Z}$ & $\mathrm{p}$ & $\mathrm{R}^{2}$ \\
\hline $\mathrm{H} 2$ & $\begin{array}{c}\text { Atratividade } \rightarrow \\
\text { Compra }\end{array}$ & 0,498 & 0,049 & 12,120 & $* * *$ & \multirow{4}{*}{0,89} \\
\hline H3 & $\begin{aligned} & \text { Impulsividade } \rightarrow \text { Intenção de } \\
& \text { Compra }\end{aligned}$ & 0,135 & 0,066 & 3,567 & $* * *$ & \\
\hline $\mathrm{H} 4$ & $\begin{aligned} & \text { P. Hedônica } \rightarrow \text { Intenção de } \\
& \text { Compra }\end{aligned}$ & 0,178 & 0,051 & 4,783 & $* * *$ & \\
\hline H5 & $\begin{aligned} & \text { R. Financeiro } \rightarrow \begin{array}{l}\text { Intenção de } \\
\text { Compra }\end{array} \\
&\end{aligned}$ & $-0,081$ & 0,043 & $-2,165$ & $* *$ & \\
\hline
\end{tabular}

Fonte: Dados da pesquisa $(* * *<0,001 ; * *<0,05)$ 
Analisando os resultados apresentados na tabela 2, identificam-se relações significativas para o desvio padrão e teste $\mathrm{Z}$ do modelo integrado. $\mathrm{O}$ coeficiente de explicação $\left(\mathrm{R}^{2}\right)$ da intenção de compra do produto em desconto foi de $89 \%$. Identifica-se ainda, uma relação positiva entre a "atratividade da promoção" e a "intenção de compra do produto em desconto" (hipótese 2); assim como entre a "impulsividade" e "intenção de compra do produto em desconto" (hipótese 3); e, ainda, entre "percepção hedônica" e "intenção de compra do produto em desconto" (hipótese 4). Encontra-se, também, uma confirmação para a hipótese 5 , já que era prevista (e foi confirmada) a relação negativa entre "risco financeiro" e "intenção de compra do produto em desconto". A seguir serão apresentadas as principais conclusões do estudo, contribuições acadêmicas e gerenciais, assim como limitações e recomendações para futuros estudos. Salienta-se que foram inclú́das no modelo final testado as seguintes covariáveis: renda, sexo e idade, sendo que nenhuma delas obteve relação significativa com a intenção de compra do produto $\left(\beta_{\text {renda }}=-, 028\right.$; $\left.\beta_{\text {sexo }}=-, 009 ; \beta_{\text {idade }}=--, 010\right)$, indicando, desta forma, que estes elementos não influenciaram no resultado e, portanto, não interferiram como varáveis estranha no estudo.

\section{Discussão dos resultados}

O presente trabalho objetivou preencher lacunas acadêmicas e teóricas sobre o entendimento das ações de promoção de vendas e seus efeitos sobre o comportamento do consumidor. Para isto, conforme destacado, realizou-se uma pesquisa experimental de laboratório onde foi possível avaliar, primeiramente, a influência da promoção de vendas de desconto na intenção de compra do consumidor. Em seguida, analisou-se o impacto da atratividade, impulsividade, percepção hedônica e de risco financeiro sobre a intenção de compra de um produto em desconto.

Os resultados, num primeiro momento, ratificaram a suposição de que a promoção de desconto interfere positivamente na intenção de compra sobre um produto (H1). Este dado consolida pesquisas anteriores, que já demonstravam o efeito positivo da promoção de vendas no comportamento do consumidor (GUPTA, 1988; ALVAREZ; CASIELLES, 2005; BOSCHET'TI, 2012). Reforçam-se, ainda, as suposições teóricas de que a promoção de vendas é um elemento importante para influenciar o comportamento de consumo (BLATTBERG; NESLIN, 1990), estimular demanda de compra (AMA, 2007) e encorajar troca de marca (GUPTA, 1988; DAVIS; INMAN; MCALISTER, 1992).

No âmbito gerencial, os resultados reforçam elementos importantes no gerenciamento de estoques, por apresentarem, mais uma vez, que a promoção de desconto pode ser um elemento primordial para o estímulo de vendas de produtos estocados e, ainda, uma ferramenta de diferenciação em categorias de produtos de alta competitividade (JONES, 2008). Além disso, as empresas podem vislumbrar esta ferramenta como um estímulo de experimentação de novos produtos (NBUDISI; MOI, 2005), assim como de aumento do volume de vendas em curto prazo (BLATTTBERG; NESLIN, 1990).

Na segunda hipótese, previa-se uma relação positiva entre a atratividade da promoção e a intenção de compra do produto em desconto. Mais uma vez, os resultados dão sustentação à proposição levantada, o que vem consolidar resultados encontrados em pesquisas prévias (SIMONSON; CARMON; O'CURRY, 1994; D'ASTOUS; LANDREVILLE, 2003; LIAO, 2006). Em termos gerenciais, estes resultados contribuem para a premissa de que o benefício ofertado deve ser atrativo, elemento este que poderá ser decisivo como um fator de sucesso ou fracasso de uma ação promocional (SIMONSON; CARMON; O'CURRY, 1994; D'ASTOUS; LANDREVILLE, 2003; LIAO, 2006), assim como para uma avaliação positiva do produto ou marca ofertada (D'ASTOUS; JACOB, 2002; D'ASTOUS; LANDREVILLE, 2003).

Na sequência, a terceira hipótese sustentou uma relação positiva entre a impulsividade do consumidor e a propensão para comprar um produto em desconto. No contexto da academia, esta confirmação contribui para reforçar teorias que sugerem a dificuldade do controle impulsivo sob a relutância de postergar gratificações que, neste caso, são ilustradas pela promoção de desconto (ROOK, 1987, COBB; HOYER, 1986; ROOK; FISHER, 1995; PURI, 1996; HAUSMAN, 2000; RAMANATHAN; MENON, 2006). Além disso, os resultados apresentados reforçam pesquisas anteriores. que relacionavam diferentes técnicas de promoção de vendas ao estímulo para a realização de compras por impulso (LIAO; SHEN; CHU, 2009).

No âmbito gerencial, o resultado ratifica a pesquisa de Mattson e Dubinsky (1987), a qual sugere que 
fatores situacionais podem representar resultados significativos no comportamento impulsivo de compra. Sugere-se, também, tomando como base a reflexão de Rook e Fischer (1995), que grande parte dos consumidores é impulsiva, que a ação de desconto poderá ser um estimulador do aumento de vendas, uma vez que pessoas com características impulsivas têm maior sensibilidade a receber uma recompensa, ilustrada, neste caso, pela promoção de desconto (RAMANATHAN; MENON, 2006).

A quarta hipótese propusera uma relação positiva entre a percepção hedônica do produto ofertado e a intenção de comprar este bem em desconto. Relação esta novamente confirmada. Reforça-se, a partir do resultado, a suposição de que a promoção de desconto possa ser um indutor para a busca de gratificações pessoais (FORSYTHE; BUTLER; KIM,1991; BABIN; DARDEN; GRIFFIN, 1994), excitação (HIRSCHMAN; HOLDBROOK, 1982; SPANGENBERG; VOSS; CROWLEY, 1997) e realização de compras não planejadas (BATRA; AHTOLA, 1990; RAMANATHAN; MENON, 2006; CARDOSO; PINTO, 2010), cujas características se associam com a aquisição de um produto hedônico (KIVETZ; SIMONSON, 2002; KWOK; UNCLES, 2005).

No âmbito gerencial, o resultado pode ser útil, na medida em que os descontos utilizados para produtos com características mais hedônicas podem provocar maior excitação (HIRSCHMAN; HOLBROOK, 1982). Outra suposição interessante está vinculada à utilização desta ferramenta para lançamento de novos produtos, haja vista que este sentimento está relacionado à percepção hedônica (VENKATRAMAN; PRICE, 1990; KIM, 2008) e os resultados demonstraram relações positivas desta percepção com a intenção de compra do produto em desconto.

Por fim, a quinta hipótese previa uma relação negativa entre a percepção de risco financeiro e a intenção de compra do produto em desconto, o que novamente foi confirmado. Diante do resultado apresentado, fortalece-se o conceito acerca do medo de perda monetária vinculado ao sentimento da percepção de risco financeiro, mesmo sob os efeitos do desconto (JACOBY; KAPLAN, 1972; DHOLAKIA, 2000; HUANG; SCHRANK; DUBINSKY, 2004).

Para os gestores, é interessante esta constatação, no sentido de pensarem alternativas para além da promoção de desconto, as quais possam vir a ser minimizadoras de uma percepção negativa de risco financeiro. Alternativas, talvez, pautadas em características do desempenho do produto ou na construção e reforço de marca, e não somente em ações de curto prazo, cujo objetivo seja a venda, como é o caso das campanhas de descontos (GUPTA, 1988; ALVAREZ; CASIELLES, 2005).

\section{Considerações finais}

O estudo aqui proposto procurou contribuir para um melhor entendimento a respeito de comportamentos relacionados à promoção de vendas e sua relação com a intenção de compra do consumidor. Entre as implicações da presente pesquisa destacam-se a reafirmação empírica da eficácia da promoção de desconto no aumento da intenção de compra em consumidores brasileiros. Demonstrou-se também a interveniência simultânea de quatro variáveis na intenção de compra de um produto com desconto que podem auxiliar pesquisadores e gestores na busca do entendimento das variáveis intervenientes em uma promoção de desconto, tanto para buscar o entendimento dessas variáveis quanto na busca da eficácia da promoção.

Com esses resultados é possível entender que para aumentar a eficácia de uma promoção de desconto, do ponto de vista do ofertante, é importante reforçar a atratividade da promoção e da percepção hedônica do produto. Além disso, entende-se que ao estimular reações mais impulsivas obtem-se melhores resultados com a promoção de desconto. Complementando essas afirmações se o consumidor não perceber risco financeiro, ou se esse for baixo, ao comprar um produto com desconto a possibilidade que efetive a compra é maior.

De uma forma geral, o presente trabalho traz constatações interessantes para serem refletidas, especialmente no contexto do varejo, uma vez que as técnicas de promoção de vendas e, essencialmente, as ações de desconto são amplamente utilizadas neste ambiente (GILBERT; JACKARIA; 2002; OH; KNOW, 2008; TONG; LAI; TONG, 2011), haja visto o número de publicações predominantes nessa área e os comportamentos encontrados no dia a dia do varejo. O tema "promoção de vendas" e sua investigação ainda possuem um amplo campo para debates, pois, apesar de ser uma técnica bastante utilizada e considerada no campo gerencial, é um 
assunto pouco analisado no âmbito acadêmico (D'ASTOUS; LANDREVILLE, 2003; ALVAREZ; CASIELLES, 2005; JONES, 2008), especialmente no Brasil (SANTINI, 2013).

No contexto nacional a ocorrência de pesquisas sobre o tema ainda á um desafio e ampliar os estudos pode trazer, além de contribuições acadêmicas, contribuições para os varejistas brasileiros compreenderem melhor que tipo de promoção de vendas sensibiliza mais o consumidor brasileiro. Nesse sentido, a promoção de desconto torna-se especialmente atraente para o consumidor oferecendo uma vantagem tangível na aquisição de determinado produto que combinada aos achados da pesquisa poderá trazer para os gestores importante ferramenta para a eficaz alavancagem de vendas. Embora os resultados tenham contribuições acadêmicas e gerenciais importantes o estudo encontra limitações, detectadas ao longo do trabalho.

\section{Limitações e estudos futuros}

As limitações que aqui podem ser destacadas dizem respeito à técnica de amostragem utilizada, ao público ao qual a pesquisa foi aplicada e, ainda, à característica do experimento utilizado. Pesquisas posteriores poderão utilizar amostras com características diferentes, para ratificar ou não os resultados encontrados nesta pesquisa. Interessante, também, seria pensar na possibilidade de empresas aplicarem pesquisas como esta aos seus clientes. A técnica de amostragem utilizada, não probabilística, também não permite atribuir os resultados de forma generalizada dentro da população investigada. Destaca-se também como fator limitador a não mensuração dos respondentes a respeito do conhecimento do produto objeto de investigação. Assim, novas pesquisas poderão contemplar esta mensuração para avaliar se este comportamento interfere nos resultados aqui analisados.

Sendo assim, pode-se sugerir a aplicação de pesquisas com técnicas de amostragem probabilística, permitindo generalização para a amostra utilizada. Quanto à característica do experimento utilizado, mesmo com as justificativas apresentadas no capítulo de metodologia, admite-se que ele tem limitações para confirmar a validade externa da pesquisa. Por esta razão, é de bom senso sugerir e realizar pesquisas que contemplem experimentos de campo para ratificar ou não os resultados aqui detectados.

\section{Referências}

AAKER, D. A. (1991) Managing Brand Equity: Capitalizing on the Value of a Brand Name. New York: The Free Press.

ALVAREZ, B; CASIELLES, R.V. (2005) Consumer Evaluations of Sales Promotion: the Effect on Brand Choice. European Journal of Marketing, v.39, n. 1/2, pp. 54-70.

APAYDIN, F; KÖKSAL, M.E. (2011) Turkish Consumers' Risk Perception Towards Global Computer Brands. International Journal of Marketing Studies, v. 3, n. 3, pp. 165-173.

ARBUCKLE, J. L. (2008) Amos 17 user's guide. Chicago, IL, SPSS.

BABIN, B.J; DARDEN, W.R; GRIFFIN, M. (1994) Work and/or Fun? Measuring Hedonic and Utilitarian Shopping Value.Journal of Consumer Research, v. 20, pp. 644-656.

BATRA, R; AHTOLA, O.T. (1990) Measuring the Hedonic and Utilitarian Sources of Consumer Attitudes. Marketing Letters, v. 2, n. 2, p. 159-170.

BAWA, K; SHOEMAKER, R.W. (1987) The Effects of a Direct Mail Coupon on Brand Choice Behavior. Journal of Marketing Research, v. 24, pp. 370-376.

BENTLER, P.M. (1995) EQS structural equations program manual. Encino, CA: Multivariate Software.

BLATTBERG, R.C.; NESLIN, S. (1990) Sales Promotion Concepts, Methods, and Strategies, New Jersey: Prentice-Hall.

BOSCHETI, R. B. (2012) Promoção de Vendas não monetária e seus efeitos na intenção de compra e na escolha da marca de serviços financeiros. Dissertação (Mestrado). Pontifica Universidade Católica do Rio Grande do Sul.

BRUNER, G.C. I.; HENSEL, P.J. (1998) Marketing Scales Handbook: a Compilation of Multi-items Measures. Chicago: American Marketing Association, v. 2 .

CALDER, B.J.; PHILIPS, L.W.; TYBOUT, A.M. (1981) Designing Research for Application. Journal of Consumer Research, v. 8, n. 2, pp. 197-207. 
CARDOSO; P.R.; PINTO, S.C. (2010) Hedonic and Utilitarian Shopping Motivations Among Portuguese Young adult Consumers. International Journal of Retail \& Distribution Management, v. 38, n. 7, pp. 538-448.

CHANDON, P.; WANSINK, B; LAURENT, G.A. (2000) Benefit Congruency Framework of Sales Promotion Effectiveness. Journal of Marketing, v.64, n. 4, pp. 65-81.

CHURCHILL JR., G.A. (1999) Marketing Research: Methodological Foundations. Orlando: The Dryden Press.

COLEY, A.; BURGESS, B. (2003) Gender Differences in Cognitive and Affective Impulsive Buying. Journal of Fashion Marketing and Management, v. 7, n. 3, pp. 282-295.

CONLON, T.J. (1980) Sweepstakes Rank as Tops. Advertising Age, p. 56-8, 6 oct.

D’ASTOUS; A; JACOB, I. (2002) Understanding Consumers Reactions to Premium-based Promotional Offers. European Journal of Marketing, v. 36, n. 11, pp. 1270-86.

D'ASTOUS, A.; LANDREVILLE, L. (2003) An Experimental Investigation of Factors Affecting Consumers' Perceptions of Sales Promotions. European Journal of Marketing, v.37, n. 11/12, pp. 1746.

DAHR, R.; WERTENBROCH, K. (2000) Customer Choice Between Hedonic and Utilitarian Goods. Journal of Marketing Research, v. 37, n. 1, pp. 60-71.

DAVIS, S.; INMAN, J.J.; MCALISTER, L. (1992) Promotion has a Negative Effect on Brand Evaluations- or does it? Additional Disconfirming Evidence. Journal of Marketing Research, v. 24, pp.143-148.

DIACON, S. (2004) Investment Risk Perceptions: Do Consumers and Advisers Agree? The International Journal of Bank Marketing, v. 22, n. 3, pp. 180-198.

DHOLAKIA, U.M. (2000) Temptation and Resistance: an Integrated Model of Consumption Impulse Formation and Enactment. Psychology and Marketing, v. 17, n. 11, pp. 955-982.
ENGELBERG, E. (2007) The Perception of Selfefficacy in Coping with Economic Risks Among Young Adults: an Application of Psychological Theory and Research. International Journal of Consumer Studies, v. 31, pp. 95-101.

GARRETSON, J. A.; BURTON, S.; CLOW, K.E. (1999) The Influence of Coupon Face Value on Service Quality Expectations, Risk Perceptions and Purchase Intentions in the Dental Industry. The Journal of Services Marketing, v. 13, n. 1, pp. 59-72.

GUPTA, S. (1988) Impact of Sales Promotions on When, What, and How Much to Buy. Journal of Marketing Research, v. 25, pp. 342-355.

HAANS, H.; GIJSBRECHTS, E. (2011) “One-dealfits-all?” On Category Sales Promotion Effectiveness in Smaller Versus Larger Supermarkets. Journal of Retailing, v. 87, n. 4, pp. 427-443.

HAIR J. R. et al. (2005) Análise Multivariada de Dados. 5.ed. Porto Alegre: Bookman.

HAUSMAN, A.A (2000) Multi-method Investigation of Consumer Motivations in Impulse Buying Behavior. Journal of Consumer Marketing, v. 17, pp. 403-419.

HISRICH，R.; DORNOFF，R.J.; KERNAN, J.B. Perceived Risk in Store Selection. Journal of Marketing Research, v. 9, pp. 435-439, 1972.

HIRSCHMAN, E.C.; HOLBROOK, M.B. (1982) Hedonic Consumption: Emerging Concepts, Methods and Propositions. Journal of Marketing, v. 46, pp. 92-107.

HOCH, S.J.; LOEWENSTEIN, G. (1991) Timeinconsistent Preferences and Consumer Self-control. Journal of Consumer Research, v. 17, pp. 492-507.

HUANG, W-Y; SCHRANK, H.; DUBINSKY, A.J. (2004) Effect of Brand Name on Consumers' Risk Perceptions of online Shopping. Journal of Consumer Behaviour, v. 4, n. 1, pp. 40-50.

INMAN, J.J.; MCALISTER, L.; HOYER, W.D. (1990) Promotion Signal: Proxy for a Price Cut? Journal of Consumer Research, v. 17, pp. 74-81.

IYER, E. (1989) Unplanned Purchasing: Knowledge of Shopping Environment and Time Pressure. Journal of Retailing, v. 65, pp. 40-57. 
JACOBY, J.; KAPLAN, L.B. (1972) The Components of Perceived Risk. In: Annual Conference, 3., Association for Consumer Research, Proceedings of... Chicago, 1972. pp. 382-393.

JAGODA, D. (1984) Sweepstakes: It's not what you give But what you Get. Marketing Communications, v. 49 , pp. 27-31.

JASPER, C.R.; OUELLETTE, S.J. (1994) Consumers' Perception of Risk and the Purchase of Apparel from Catalog. Journal of Direct Marketing, v. 8 n. 2, pp. 23-26.

JONES, J.M. (2008) An Exploratory Study on Attitude Persistence Using Sales Promotion. Journal of Managerial Issues, v. 20, n. 3, pp. 401-416.

KELLER, K.L. (1993) Conceptualizing, Measuring, and Managing Customer-based Brand Equity. Journal of Marketing, v. 57, n. 1, pp. 249-66.

KELLER, K.L.; LEHMANN, D.R. (2006) Brands and Branding: Research Findings and Future Priorities. Marketing Science. v. 1, n. 6, pp. 740-759.

KEPPEL, G. (1991) Design and Analysis: a Researcher's Handbook. Upper Saddle River: Prentice Hall.

KLINE, R. (1998) Principles and practice in structural equation modeling. New York: Guilford Press.

KWOK, S.; UNCLES, M. (2005) Sales Promotion Effectiveness: the Impact of Consumer Differences at an Ethnic-group Level. The Journal of Product and Brand Management, v.14, n. 2/3, pp. 170-186.

LAROCHE, M. et al. (2003) A Model of Consumer Response to Two Retail Sales Promotion Techniques. Journal of Business Research, v. 56, pp. 513-522, 2003.

LATTIN, J. M.; BUCKLIN, R. E. (1989) Reference Effects of Price and Promotion on Brand Choice Behavior. Journal of Marketing Research, v. 26, pp. 299-310.

LEE, C.W. (2002) Sales Promotions as Strategic Communication: the Case of Singapore. The Journal of Product and Brand Management, v. 11, n. 2/3, pp. 103-114.
LIAO, S. (2006) The Effects of Nonmonetary Sales Promotions on Consumer Preferences: The Contingent Role of Product Category. Journal of American Academy of Business, Cambridge, v. 8, n. 2, p. 196.

MALHOTRA, N. K. (2006) Pesquisa de Marketing. Porto Alegre: Bookman.

MALHOTRA, N. K. (2012) Pesquisa de Marketing. Porto Alegre: Bookman.

MANN, P. S. (2006) Introdução à estatística. 5. ed. Rio de Janeiro: LTC.

MARÔCO,J. (2010) Análise de equações estruturais - Fundamentos teóricos, software e aplicações. Pero Pinheiro (Portugal): Report Number.

MARDIA, K.V. (1970) Measures of multivariate skewness and kurtosis with applications. Biometrika, 36, pp. 519-530.

MITCHELL, V.W. (1999) Consumer Perceived Risk: Conceptualizations and Models. European Journal of Marketing, v. 33, pp. 163-195.

NIJS, V.R. et al. (2001) The Category demand Effects of Price Promotions. Marketing Science, v. 20, n. 1, pp. 1-22.

OFIR, C.; BECHTEL, G. (1990) Scaling and Dimensionalizing Perceived Risk from Ratings Data: Managers' Risk Perception of Business Computers. Marketing Letters, v. 1, n. 2, pp. 171-179.

PALAZON, M.; DELGADO-BALLESTER, E. (2009) Effectiveness of Price Discounts and Premium Promotion. Psychology \& Marketing, v. 26, n. 12, pp. 1108-1129.

PETERSON, R. (2001) On the Use of College Students in Social Science and Research: Insights from a Second-order meta-analysis. Journal of Consumer Research, v. 28, n.3, pp. 450-461.

PRESTON, RH, DWYER, F., AND RUDELIUS, W., "The Effectiveness of Bank Premiums," Journal of Marketing, 1978, 42, (3), 96-101.

PURI, R. (1996) Measuring and Modifying Consumer Impulsiveness: a Cost-benefit Accessibility Framework. Journal of Consumer Psychology, v. 5, n. 2 , pp. $87-113$. 
RAMANATHAN，S.; MENON，G. (2006) TimeVarying Effects of Chronic Hedonic Goals on Impulsive Behavior. Journal of Marketing Research, v. 43, n. 4 , pp. 628-641.

ROOK, D.W. (1987) The Buying Impulse. Journal of Consumer Research, v. 14, pp.189-199.

ROOK, D.W.; FISHER, R. (1995) Normative Influences on Impulsive Buying Behavior. Journal of Consumer Research, v. 22, pp. 305-313.

ROOK, D.W.; GARDNER, M.P. (1993) In the Mood: Impulse Buyings' Affective Antecedents. In: ArnoldCosta, Janeen; Belk, Russell W. (Eds.). Research in Consumer Behavior, 6., Greenwich, CT: JAI Press, pp. 1-28.

SANTINI, F. O. (2013) Uma análise da influência da promoção de vendas de desconto na intenção de compra do consumidor e os efeitos moderadores da atratividade. Tese de Doutorado, Programa de PósGraduação em Administração, Pontifícia Universidade Católica do Rio Grande do Sul.

SCHINDLER, R.M. (1992) A Coupon is More than a Low Price: Evidence from a Shopping-simulation Study. Psychology \& Marketing, v. 9, n. 6, pp. 431451.

SIMONSON, I.; CARMON, Z.; O'CURRY, S. (1994) Experimental Evidence on the Negative Effect of Product Features and Sales Promotion on Brand Choice. Marketing Science, v. 13, pp.23-40.

SIMON, S. M.; VICTOR, T. F. (1994) Customers' Risk Perceptions of Electronic Payment Systems. International Journal of Bank Marketing, v. 12, n. 8, pp. 26-38.

SITKIN, S.B.; WEINGART, L.R. (1995) Determinants of Risky Decision-making Behavior: a Test of Mediating Role of Risk Perception and Propensity. Academy of Management Journal, v. 38, n. 6, pp. 1573-1592.

SPANGENBERG, E.R.; VOSS, K.E.; CROWLEY, A.E. (1997) Measuring the Hedonic and Utilitarian Dimensions of Attitude: a Generally Applicable Scale". Advances in Consumer Research, v. 24, n. 1, pp. 235-41.

STONE; R.N.; GRØNHAUG, K.(1993) Perceived risk: Further considerations for the marketing discipline. European Journal of Marketing, v. 27, n.3, pp. 3950.
TAYLOR, G.A.; NESLIN, S.A. (2005) The Current and Future Sales Impact of a Retail Frequency Reward Program. Journal of Retailing, v. 81, n. 4, pp. 293 305.

TEUNTER, L.H. (2002)Analysis of sales promotion effects on household purchasing behavior. ERIM $\mathrm{PhD}$ Research Series in Management. Erasmus University Rotterdam, pp. 262-272.

URDAN, F.T; URDAN, A.T. (2001) O impacto da marca sobre as preferências do consumidor: um experimento com cervejas. In: XXV Encontro Anual da ANPAD, 2001, Campinas. Anais.

WEEGELS, M.F.; KANIS, H. (2000) Risk Perception in Consumer Product Use. Accident Analysis and Prevention, v. 32, n. 3, pp. 365-370.

WEINBERG, P.; GOTTWALD, W. (1982) Impulsive Consumer Buying as a Result of Emotions. Journal of Business Research, v. 10, pp. 43-57.

WESTBROOK, R.; BLACK, W. (1985) A Motivationbased Shopper Typology. Journal of Retailing, v. 61, pp. $78-103$.

WIERENGA, B.; SOETHOUDT, H. (2010) Sales Promotions and Channel Coordination. Journal of the Academy of Marketing Science, v. 38, n.3 pp. 383-397.

WILSON, T.D.; ARONSON, E.; CARLSMITH, K. (2010) The art of laboratory experiment. In: FISKE, S.; GILBERT, D.; LINDZEY, G. The Handbook of social psychology, pp.49-79.

WINER, R.A. (1986) Reference Price of Brand Choice for Frequently Purchased Products. Journal of Consumer Research, v. 13, pp. 250-256.

YOUN, S. (2000) The Dimensional Structure of Consumer buying Impulsivity: Measurement and Validation. [S.1.], 2007. Tese (Doutorado em Administração) - University of Minnessota, 2000.

YOUN, S.; FABER, R. (2000) Impulsive Buying: its Relation to Personality Traits and Cues. Advances in Consumer Research, v. 27, pp. 179-185.

YUSUF, J. B. (2010) Ethical Implications of Sales Promotion in Ghana: Islamic Perspective. Journal of Islamic Marketing, v. 1, n. 3, pp. 220-230. 